\title{
Introduction to special issue on body representation: feeling, seeing, moving and observing
}

\author{
Ellen Poliakoff
}

Published online: 17 June 2010

(c) Springer-Verlag 2010

'All the real or imaginary symptoms of his older patients made their appearance in his body. He felt the shape of his liver with such clarity that he could tell its size without touching it. He felt the dozing cat's purr of his kidneys, he felt the iridescent brilliance of his vesicles, he felt the humming blood in his arteries.'

\section{Gabriel García Márquez, Love in the Time of Cholera}

Since Berlucchi and Aglioti's seminal review 'The Body in the Brain' in 1997, research into the representation of the body has expanded and new experimental paradigms and approaches have been developed. This special issue of Experimental Brain Research has emerged from a series of workshops on Body Representation over the past 4 years, which have taken a broad view of what constitutes body representation. This breadth reflects the multiple purposes and effects of representing one's own body, and those of other people. To illustrate this, the quote above describes how a doctor's perception of his own body is influenced not just by current sensory input, but also by his knowledge of his own body and the symptoms he has observed in other people.

\section{Needing a body of knowledge}

Our perception and ongoing knowledge of our bodies comes from different receptors and sensory modalities, which is dealt with by a number of papers in this issue. Many aspects of body perception are essential for, and inti-

E. Poliakoff $(\bowtie)$

School of Psychological Sciences, The University of Manchester,

Zochonis Building, Brunswick Street, Oxford Road,

Manchester M13 9PL, UK

e-mail: Ellen.Poliakoff@manchester.ac.uk mately connected, with action, enabling us to interact with other people and objects in our environment by approaching or avoiding them. In keeping with the dual-route visual pathway, a distinction has been drawn between touch for perception versus touch for action (Dijkerman and de Haan 2007). The distinction has also been made between the body as an instrument for action, versus an object of perception (Gallagher 2005).

Often when we perceive objects through touch, they come into contact with separate areas of skin. It has been found that if two adjacent fingers are stroked in synchrony with wooden rods, people integrate the two inputs, perceiving that they are being stroked by a single object (Kitada et al. 2003). Following this work, Peelen et al. (this issue) show that this integration still occurs when stroking a finger on each hand or when two toes are stroked. This perceptual integration process was associated with a network of activity across the right inferior parietal lobule, the left inferior temporal sulcus and the left middle frontal gyrus (see also Kitada et al. 2003). Interestingly, integration across the body midline produced differential activation of the left intraparietal sulcus, an area already implicated more generally in the binding together of sensory inputs.

Proprioception, the ability to localise our bodies and limbs in space, is critical for perception and action. Jones et al. (this issue) found that when judging the location of their hand relative to the midline, people tended to perceive their left hand as further to the left and their right hand as further to the right. This may reflect a fundamental misperception of hand position, which warrants further investigation.

Vision can also influence perception of the body and touch. This can be vision of the body itself (Kennett et al. 2001) or visual stimuli presented close to the body. For example, in the crossmodal congruency task developed by 
Spence et al. visual stimuli presented close to the body can influence judgements about tactile stimuli presented at the same time (for a review, see Maravita et al. 2003). In an extension of this work, Igarashi et al. (this issue) showed that spatial judgements of touches to the forefinger (tip versus base) were influenced by visual distractors positioned in front of participants, even when there was no obvious spatial relationship with the finger. This interference effect was affected by the posture of their (hidden) hand, but the effects were always high for two visual orientations; vertical and $45^{\circ}$ towards the midline. The authors suggest that this involves off-line knowledge of typical postures of the hand, as well as knowledge about current hand posture.

Preparing, or being about to perform, an action has been found to suppress somatosensory perception (Chapman et al. 1988). Walsh and Haggard (this issue) used this effect to probe the effects of motor inhibition. They found that even when an attempt to inhibit a pre-prepared action was unsuccessful, there was still a brief attenuation of this somatosensory suppression. This shows that motor inhibition can exert a sensory effect even when the inhibition of the motor response ultimately fails. This demonstrates the flexible interplay between somatosensory and motor processes.

Another, often neglected aspect of touch and the body is its social function, which is addressed in a timely review by Morrison et al. (this issue). Pleasant touch can increase interactions between people (affiliative behaviour), produce and support social bonds as well as allowing emotions to be communicated nonverbally. They link these functions to recent discoveries about the affective processing of touch, from the skin to the brain.

In terms of identifying different types of body representation, dissociations in the effect of bodily illusions on different perceptual and motor tasks have been used to argue for an ever growing number of putative body representations. Kammers et al. (this issue) caution against this approach and suggest a way forward. They argue, and demonstrate, that considering the differential weighting of information can explain such findings without recourse to expanding the number of body representations.

\section{Whose body is it anyway?}

How do we know that our body is our own? As mentioned above, the fact that we can simultaneously feel and see our own body is one of its defining characteristics. A very influential line of work has involved the rubber hand illusion. Botvinick and Cohen (1998) observed that stroking a rubber hand at the same time as a person's hidden real hand led people to perceive their hand in a similar position as the seen fake hand, and to feel some sense of ownership over this rubber hand. Since this initial observation, there has been much research into the boundary conditions for this illusion and it has been suggested that we might take ownership of any object stroked synchronously with our body (Armel and Ramachandran 2003). However, Tsakiris et al. (this issue) demonstrated that although people were sensitive to the synchrony of stroking of non-body objects, it was only for the plausibly shaped rubber hand that synchrony produced changes in self reports and localisation. For this to occur, they suggest that an object must match our implicit knowledge of the structure of our body (see also Igarashi et al. above). Indeed, de Preester and Tsakiris (2009) argue that this knowledge and resultant ownership distinguish the incorporation of artificial hands or prosthetics into the body from the extension of the body with tools.

Newport, Pearce and Preston (this issue) explored the effect of adding an action component to the fake hand illusion. In a novel set-up, using real-time manipulated visual feedback, participants observed a virtual image of their own hand displaced in space to the left or right of their real hand. If one hand moved synchronously with their real hand, their reported ownership of that virtual hand was higher and they reached as if their real hand were in the location of the synchronous virtual hand. When both fake hands moved in synchrony, participants reported feeling ownership of both hands, creating an analogue of the phenomenon of supernumerary limbs where patients feel that they have more than one of a particular limb. However, they still reached as if controlling a single hand, perhaps reflecting how action constrains ownership.

There is also something distinctive about observing the body of another person. Two brain areas have been found to respond preferentially to bodies (without faces) as compared to other objects; the extrastriate body area (EBA, Downing et al. 2001) and the fusiform body area (FBA, Peelen and Downing 2005). While these areas respond to static bodies, there has been a great deal more research on the observation of other people's actions. Much of this research has been inspired by the report of mirror neurones in the primate brain that respond to both the monkeys' own action and the sight of another person moving (di Pellegrino et al. 1992).

Cross et al. (this issue) investigated how the EBA and FBA respond to contorted versus normal body postures. Both areas responded more strongly to contorted body postures compared to ordinary postures, but the FBA also showed a viewpoint independent response to body postures. This suggests a way of separating the roles of the EBA and FBA. A further set of areas, more commonly associated with the observation of other people's actions, also showed a viewpoint independent response. This implies that viewing real action, or even implied action, is not required to activate this system. Investigating another aspect of body 
posture, Calvo-Merino et al. (this issue) asked participants to make aesthetic judgements about body postures. They examined the effect of transcranial magnetic stimulation (TMS) on the participant's preference between two postures and assessed whether this preference reflected the person's own ratings when shown the postures individually. This subjectivist approach did not assume which postures would be aesthetically pleasing. They observed differential effects of repetitive TMS over the EBA and the ventral premotor area, with the latter tending to improve and the former tending to worsen judgements. This therefore suggests a role for these body-related brain areas in appreciating aesthetic aspects of the body in space.

Three papers in this issue concern observation of the actions of other people for different purposes; imitation, action judgment and emotional judgment. First, Wild et al. (this issue) asked participants to observe a series of pointing movements in order to imitate the action. Unbeknownst to participants, they manipulated the speed of the movement. They found that participants modulated their own speed only when they observed the pointing action in the absence of a goal. This suggests differential mechanisms underlie the imitation of goal and goal-less actions. Second, in the study by Poliakoff et al. (this issue), participants used motion of a single hand to judge the weight being lifted (see also Hamilton et al. 2004). Finally, in a novel paradigm, McHugh et al. (this issue) asked participants to judge the emotion of a crowd of bodies. They found that the dynamic bodily movements of individuals within the crowd were an efficient cue for perceiving the emotion of the crowd.

Viewing another person's action can involve the observer's own somatosensory areas as well as motor areas (Gazzola and Keysers 2009). Furthermore, if we view another person's body being touched this can activate our own somatosensory areas (Keysers et al. 2004). Morrison et al. (this issue) review this intersubjective representation of touch and pain. While they suggest that this underlies empathy and the perception of others' emotions, they propose that empathy may be secondary to learning about typical contexts of touch and pain, rather than the other way round.

\section{Differing bodies}

An important facet of body representation is the differences between people. This encompasses changes in representation of the body in patient populations, as well as individual differences in the general population. For example, Burrack and Brugger (2005) have shown individual differences in the experience of illusory arm extension in response to tendon vibration of the arm. In the current issue, Cadieux et al. investigated whether there were gender differences in the detrimental effect of crossing one's hands on tactile temporal order judgments. The crossed hands effect has been attributed to the use of a visual frame of reference, since the congenital blind do not show this effect (Röder et al. 2004). Cadieux et al. found that females were more impaired by crossing their hands. Females also showed a stronger effect of a visual frame when judging the verticality of a line (relative to gravity), suggesting that a stronger reliance on the visual frame of reference may underlie the crossed hands effect. This study highlights the importance of considering individual differences in future studies of body perception and misperception, not captured by averaging data. The subjectivist approach of Calvo-Merino et al. (this issue) provides another strategy for building individual differences into research.

A key feature of the representation of our body is that even after development in infancy (Bremner et al. 2008), it is flexible enough to accommodate changes in size and experience over our lives (Newport et al. this issue). For example, increased finger representation in $\mathrm{S} 1$ has been observed in musicians with extensive experience with stringed instruments (Elbert et al. 1995) and reduced S1 activation has been observed after people undergo immobilisation through wearing a cast on their arm (Lissek et al. 2009).

Four studies in this issue consider how representation of the body is affected by, or implicated in, several psychological, neurological and physical conditions. Insights gained from the general research on the body can provide frameworks and paradigms to elucidate these pathological changes and suggest possible treatments, while the knowledge gained from these conditions can in turn enhance our general understanding of the body.

Anosognosia for hemiplegia is a lack of awareness of paralysis that may follow a stroke. Jenkinson and Fotopoulou (this issue) use a motor control framework to understand this condition, reviewing evidence that such patients have intact motor intentions. Thus, their deficit may lie in detecting discrepancies between predicted and perceived sensory feedback associated with actions. This suggests that some degree of discrepancy is tolerated by the healthy system and that this is increased in patients with anosognosia. This framework may help inspire rehabilitation strategies, such as using video to allow patients to view their paralysed limb from a third person perspective.

Hilti and Brugger (this issue) review two disorders of body integrity that can be seen as opposites. First, phantom limbs in people born without a limb, a phenomenon which is important to the debate on the innateness of a body representation, since they cannot be due to memory of an intact limb. They point out, however, that innate does not mean genetically hard-wired because such individuals may have early experiences in utero (see also, Price 2006). The 
opposite pattern can be seen in people with a puzzling strong desire to have a healthy limb amputated, which they feel does not belong to them ("body integrity identity disorder"). Hilti and Brugger present evidence that there is altered multisensory integration for the affected limb in this population (Blanke et al. 2009), manifesting as a lack of 'animation'.

People with motor disorder Parkinson's disease typically have difficulty in initiating movements and producing movements of sufficient amplitude. Poliakoff et al. (this issue) showed that despite changes in their own motor systems, people with Parkinson's disease were able to judge the weight of objects lifted by another person. This suggests that either the patients were still able to simulate this relatively simple movement or they were able to use a visual compensatory strategy. Cueing can be an effective therapeutic strategy for initiating movement in people with Parkinson's (Rubenstein et al. 2002) and observation of actions could be a useful therapeutic cue.

Finally, Bultitude and Rafal (this issue) present a case study of a patient with complex regional pain syndrome following recovery from a fracture of her right hand. This patient reported changes in bodily experience prior to the onset of her painful symptoms, suggesting that altered body representation might lead to pain rather than vice versa. Their observations of two types of treatment that alter sensory motor feedback (mirror box and prism adaptation) were promising, producing improvements in her pain and range of movements within a few days.

Examining sensorimotor and multisensory bodily processes in several patient groups has been a fruitful way of understanding more about the disorder and about the representation of the healthy body. This is also an important line of work for other conditions such as eating disorders (Mussap and Salton 2006), autism (Wild et al. this issue), out of body experiences (Blanke et al. 2004) and medically unexplained symptoms, that is physical symptoms with no organic cause (Brown et al. 2007). However, there are more complex aspects of such disorders that cannot be explained by sensorimotor and multisensory impairments (Jenkinson and Fotopoulou, this issue; Hilti and Brugger, this issue).

\section{Concluding remarks}

Overall, the papers in this special issue reflect the diversity of information represented about our bodies (and those of other people), from more basic sensory and motor processes, to higher level processes such as ownership, perceiving emotions, aesthetic appreciation, imitation and social interaction. There are still many challenges for this area of research, in particular, in establishing agreement about common concepts and terminology. There are also many unanswered questions and areas just beginning to receive widespread attention by researchers, such as interoception, the perception of the inside of the body (Berlucchi and Aglioti 2010).

Acknowledgments The Body Representation workshops that gave rise to this special issue have been supported by the British Psychological Society, ESRC Research Seminar Funding ('Representations of the Body in Human Psychology'), European Science Foundation European Research Network for Investigating Human Sensorimotor Function in Health and Disease (ERNI-HSF), Manchester Neuroscience Research Institute, University of Trento Centre for Mind/Brain Sciences and Cognitive Science Laboratory, University of Oxford, University of Nottingham and University of Manchester. The workshops could not have happened without the efforts of the organising committees and local organisers: Prof Patrick Haggard, Prof Charles Spence, Prof Stephen Jackson, Dr Andrea Serino, Dr Massimiliano Zampini, Dr Francesco Pavani and Dr Nick Davis, as well as all the participants at the workshops.

\section{References}

Armel KC, Ramachandran VS (2003) Projecting sensations to external objects: evidence from skin conductance response. Proc Biol Sci 270:1499-1506

Berlucchi G, Aglioti S (2010) The body in the brain revisited. Exp Brain Res 200:25-35

Blanke O, Landis T, Spinelli L, Seeck M (2004) Out-of-body experience and autoscopy of neurological origin. Brain 127:243-258

Blanke O, Morgenthaler FD, Brugger P, Overney LS (2009) Preliminary evidence for a fronto-parietal dysfunction in able-bodied participants with a desire for limb amputation. J Neuropsychol 3:181-200

Botvinick M, Cohen J (1998) Rubber hands 'feel' touch that eyes see. Nature 391:756

Bremner AJ, Holmes N, Spence C (2008) Infants lost in (peripersonal) space? Trends Cogn Sci 12:298-305

Brown RJ, Poliakoff E, Kirkman MA (2007) Somatoform dissociation and somatosensory amplification are differentially associated with attention to the tactile modality following exposure to body-related stimuli. J Psychosom Res 62:159-165

Burrack A, Brugger P (2005) Individual differences in susceptibility to experimentally induced phantom sensations. Body Image 2:307313

Chapman CE, Jiang W, Lamarre Y (1988) Modulation of lemniscal input during conditioned arm movements in the monkey. Exp Brain Res 72:316-334

de Preester H, Tsakiris M (2009) Body-extension versus body-incorporation: Is there a need for a body-model? Phenomenol Cogn Sci 8:307-319

di Pellegrino G, Fadiga L, Fogassi L, Gallese V, Rizzolatti G (1992) Understanding motor events: a neurophysiological study. Exp Brain Res 91:176-180

Dijkerman HC, de Haan EHF (2007) Somatosensory processes subserving perception and action. Behav Brain Sci 30:189-239

Downing PE, Jiang Y, Shuman M, Kanwisher N (2001) A cortical area selective for visual processing of the human body. Science 293:2470-2473

Elbert T, Pantev C, Wienbruch C, Rockstroh B, Taub E (1995) Increased cortical representation of the fingers of the left hand in string players. Science 270:305-307

Gallagher S (2005) How the body shapes the mind. Oxford University Press, New York 
Gazzola V, Keysers C (2009) The observation and execution of actions share motor and somatosensory voxels in all tested subjects: single-subject analyses of unsmoothed fMRI data. Cereb Cortex 19:1239-1255

Hamilton A, Wolpert DM, Frith U (2004) Your own action influences another person's action. Curr Biol 14:493-498

Kennett S, Taylor-Clarke M, Haggard P (2001) Non-informative vision improves the spatial resolution of touch in humans. Curr Biol 11:1188-1191

Keysers C, Wicker B, Gazzola V, Anton J-L, Fogassi L, Gallese V (2004) A touching sight: SII/PV Activation during the observation and experience of touch. Neuron 42:335-346

Kitada R, Kochiyama T, Hashimoto T, Naito E, Matsumura M (2003) Moving tactile stimuli of fingers are integrated in the intraparietal and inferior parietal cortices. NeuroReport 14:719-724

Lissek S, Wilimzig C, Stude P, Pleger B, Kalisch T, Maier C, Peters SA, Nicolas V, Tegenthoff M, Dinse HR (2009) Immobilization impairs tactile perception and shrinks somatosensory cortical maps. Curr Biol 19:1-6
Maravita A, Spence C, Driver J (2003) Multisensory integration and the body schema: close to hand and within reach. Curr Biol 13:R531-R539

Mussap AJ, Salton N (2006) A 'rubber-hand' illusion reveals a relationship between perceptual body image and unhealthy body change. J Health Psychol 11:627-639

Peelen MV, Downing PE (2005) Selectivity for the human body in the fusiform gyrus. J Neurophysiol 93:603-608

Price EH (2006) A critical review of congenital phantom limb cases and a developmental theory for the basis of body image. Conscious Cogn 15:310-322

Röder B, Rosler F, Spence C (2004) Early vision impairs tactile perception in the blind. Curr Biol 14:121-124

Rubenstein TC, Giladi N, Hausdorff JM (2002) The power of cueing to circumvent dopamine deficits: A review of physical therapy treatment of gait disturbances in Parkinson's Disease. Mov Disord $17: 1148-1160$ 